\title{
Clinical Pharmacist-Led Medication Reconciliation Initiative at a Newly Established Hospital in Qatar: A Preliminary Report
}

\author{
Hala Sonallah', Tarek Ibrahim ${ }^{1}$, Dalia Abdul Sattar ${ }^{2}$, Dalia Ahmed ${ }^{2}$, and Mohamed Izham Mohamed Ibrahim ${ }^{3 *}$ \\ ${ }^{1}$ Clinical Pharmacist, Department of Pharmacy, Al Wakra Hospital, Qatar \\ 2Pharmacy Student, College of Pharmacy, Qatar University, Qatar \\ ${ }^{3}$ Professor of Social \& Administrative Pharmacy, College of Pharmacy, Qatar University, Qatar \\ *Corresponding author: Mohamed Izham Mohamed Ibrahim, PhD, Professor of Social \& Administrative Pharmacy, \\ College of Pharmacy, Qatar University, P.O. Box 2713, Doha, Qatar, Tel: +974-4403-5580, Fax: +974-4403-555, E-mail: \\ mohamedizham@qu.edu.qa
}

\begin{abstract}
This preliminary study was conducted to identify the types and frequency of medication discrepancies, and clinical pharmacists' interventions made upon patients' admission. Medication Reconciliation (MR) was done by clinical pharmacists routinely on inpatients from intensive care unit, medical and surgical wards at Al Wakra hospital. This retrospective study was conducted on $251 \mathrm{MR}$ forms used by the clinical pharmacists. Data analysis was carried out using SPSS version 21.0. Kolmogorov-Smirnov test was done to confirm for normality. Chi-Square, Spearman rho and Mann-Whitney $U$ tests were carried out at alpha $=0.05$. Out of the 251 forms, 232 were usable. 1640 medications were reconciled. The number of medicines reconciled ranged from 1 to 17 . The majority of the medications reconciled were cardiovascular medications ( $n=144 ; 62.2 \%$ ). The highest number of medicines discrepancies were 2 with 39 cases $(16.7 \%)$ and mostly were due to medication omissions $(n=153 ; 66.1 \%)$. Clinical pharmacists intervened the highest for omitted medications that required resumption (n $=111$ of the cases; $44 \%$ ). There was a significant correlation between the percentage of discrepancies and the number of clinical pharmacists' interventions $(p<0.01)$. Gender is not a factor for number of medication discrepancies ( $p$ $=0.157)$ and the rate of omission $(p=0.281)$. Medication discrepancies upon hospital admission are highly common. The highest number of medicines discrepancies was two and mostly were due to medication omissions.
\end{abstract}

\section{Keywords}

Clinical pharmacist, Hospital pharmacy, Medication discrepancy, Medication reconciliation, Qatar

\section{Introduction}

Hospital admission is an interface of care when medication delivery becomes a complex process, and when more than half of medication errors occur [1,2] Discrepancies in medication history taking accounts for approximately $67 \%-85 \%$ of these medication errors, with a potential harm range from $11 \%$ to $59 \%$ [3-7].

Medication reconciliation is one of the best preventative strategies that has been proven to significantly reduce discrepancies and subsequent errors in relation to medication history, especially when conducted by clinical pharmacists $[2,5]$. As defined by the Joint Commission medication reconciliation is: "the process of comparing the medications a patient is taking (and should be taking) with newly ordered medications" in order to resolve discrepancies or potential problems [1].

In 2005, The Joint Commission on Accreditation of Healthcare (JCOAH) put forth medication reconciliation as National Patient Safety Goal (NPSG) in an effort to minimize adverse events caused during care transitions [5]. Since then, many international patient safety-leading organizations have been paying much attention to implement medication reconciliation in health care settings, such as Institute of Healthcare Improvement and Institute for Safe Medication Practices [5]. Moreover, both Joint Commission in the United Sates and Accreditation Canada have designated it as a required organi- 
zation practice and mandatory for accreditation $[3,4]$. Despite all of these extensive efforts, implementation of a systematic medication reconciliation process had been very challenging.

As a newest addition to Hamad Medical Corporation's (HMC) General Hospitals Group, Al Wakra Hospital (AWH), a tertiary hospital, can be a best place to implement new services, overcoming the "resistance to change" phenomenon or any other cultural and logistical barriers. However, due to the lacked of an organized medication reconciliation process like all other hospitals within HMC group, it was even more demanding for AWH to step forward establishing the formal practice of medication reconciliation and ultimately serving as a role model.

When patients are admitted to AWH hospital, physicians by policy are expected to take medication history from the patients. Many drawbacks have been observed when this method was applied. Most importantly, the accuracy and comprehensiveness of the physicians' obtained medications lists. They usually do not reflect what really the patient is taking. Without appropriate verification of the patient's medication regimen, drug-related problems may be undetected, a diagnosis may be missed, or discrepancies in patient admission orders may take place. Subsequently, adverse drug reactions are expected to occur as well as hospital readmissions, which in turn, may impact on patient safety. Physicians may perceive little value in documenting a medication history on a separate form and are not compliant with the practice of documenting the medication list within the admission history (as shown from AWH auditing medical records). Physicians' workload and constant turnover remains non-ignorable concerns that make it very difficult to adopt structured medication reconciliation processes.

As suggested by JCOAH, developing a Medication Reconciliation Form (MRF) to be used as a template for gathering information about current medications is the best strategy to standardize the care [8]. We assume that implementation of a systematic medication reconciliation process using a unified standard form will help in detecting and resolving any medication discrepancies and preventing medication errors. Being drug experts, clinical pharmacists when presenting during hospital admission, patient care rounds and when prescriptions are ordered, provide the gold standard when compiling the best possible medication history or medication reconciliation $[2,6,7]$. This study aims to evaluate clinical pharmacist-led medication reconciliation, and to identify the types, frequency of medication discrepancies and interventions made by clinical pharmacists upon patient's admission.

\section{Methods}

\section{Study design}

This was a post-interventional study conducted at AWH in Qatar that includes medical, surgical and intensive care wards between April until October 2013. Data was collected retrospectively based on the routine activities of clinical pharmacists in the hospital.

\section{Ethics approval}

An ethical approval was obtained from the institutional review board of HMC medical research center (Proposal \#13381/13; Ref \#: RC/108913/2013).

\section{Population and sampling}

The study included medication reconciliation activities conducted by clinical pharmacists on patients who were admitted in the medical, surgical and intensive care wards and followed up by the clinical pharmacists. Patient sampling was limited to those who were taking one or more regular medications during admission.

\section{Tool development and validation}

A review of English language literature was conducted in pertinent electronic health databases (i.e. Google scholar, PubMed, Science Direct) to obtain MRF samples used in other studies. The retrieved medication reconciliation samples used in other studies were evaluated and adapted by the core investigator team to design the standard form for AWH. Then the form developed for the study was content-validated. Following that, Al Wakra's clinical pharmacy division was granted the formal approval for MRF by the Pharmacy and Therapeutic committee, when it became an official document to be accessible in patients' charts. All clinical pharmacists had been using the designed form as a required task of their daily duty work for all of their patients at the first encounter on the day of admission within 24 hours. The MRF has been used to document the medication history including all the prescription medications, over the counter and herbal medications the patient has been using prior to admission. Documentation included patient's reason for admission, past medical history, medication allergy status, in addition to each medication dose, route, and frequency and indication. Sources of information used to obtain history were also included as well as any patient individual needs that might affect his/her medication use.

\section{Data collection procedure}

Clinical pharmacists then used their filled MRFs to compare the obtained list of medications with the physician admission orders and analyzing the cases order to identify any discrepancies, determine the reasons and resolve them. They also used the MRF to point out their clinical interventions in order to discuss them with the physicians.

All completed forms were included for data analysis. The form was considered complete if it included patient identification data, presenting complaint, past medical history, allergy status, medications list verification against the admission medication chart, clinical pharmacist interventions, identification of sources of information and 
patient assessment done for the listed categories in the form. Forms were excluded if they were not filled by clinical pharmacist (i.e. nurse, doctor), illegibly hand-filled, incomplete based on the mentioned predetermined criteria, or forms that contained no regular medications.

\section{Data management and analysis}

Data retrieved from the manual-filled MRFs were entered into a database using Statistical Package for Social Sciences SPSS version 21.0 software for analysis. Study results were reported as percentages of frequency or occurrence. The Kolmogorov-Smirnov test was used to assess normality of data. Chi-Square, Spearman rho and Mann-Whitney U-tests were carried out to test for correlation and differences between variables, with an alpha of less than or equal to 0.05 be considered statistically significant.

\section{Results}

A total of 232 usable MRFs were collected and 1640 medications were reconciled from 121 (52\%) male

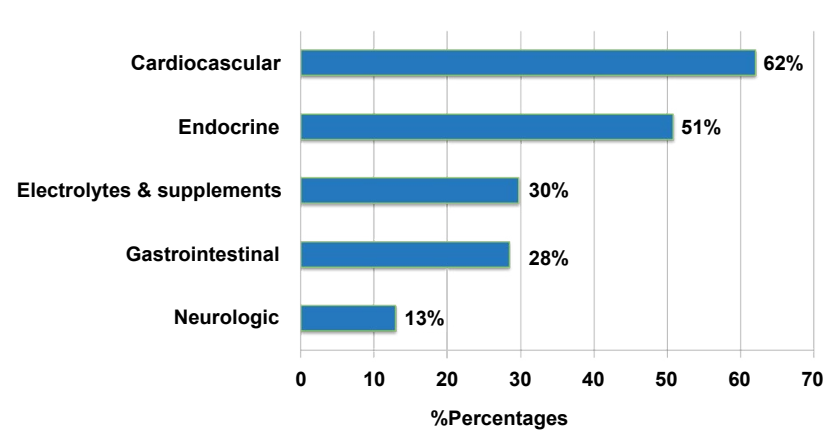

Figure 1: Most frequent classes of medications reconciled.

Table 1: Types of Medication discrepancies.

\begin{tabular}{|l|l|l|}
\hline Medication discrepancy & $\begin{array}{l}\text { \% of } \\
\text { cases }^{*}\end{array}$ & $\begin{array}{l}\text { Number of } \\
\text { occurrence (\%) }\end{array}$ \\
\hline Omission & 66.1 & $153(61.4)$ \\
\hline Different dose & 16.3 & $37(14.9)$ \\
\hline Different drug & 13.3 & $30(12.0)$ \\
\hline Frequency & 8.2 & $18(7.2)$ \\
\hline Route & 4.3 & $9(3.6)$ \\
\hline Duplication & 1.3 & $2(0.8)$ \\
\hline Total $(\mathrm{n}=232$ cases) & & $249(100)$ \\
\hline
\end{tabular}

*Note: The percentage will not add up to 100 because each case/patient might have more than one type of discrepancy. patients and 111 (48\%) female patients. Fifty-two of the patients were without errors $(25$ males and $27 \mathrm{fe}-$ males). The number of medicines reconciled by the clinical pharmacists ranged from 1 to 17 and mostly was 2 medicines ( $n=27$ cases; $11.6 \%$ ) and a median average of 6.0 (IQR: 3.0-10.8). The highest number of medicines discrepancies were 2 with 39 cases $(16.7 \%)$, followed by 1 discrepancy $(\mathrm{n}=34 ; 14.6 \%)$, then 3 discrepancies ( $\mathrm{n}$ $=23 ; 9.9 \%$ ) and the median average of medicines with discrepancies was 2.0 (IQR: 1.0-5.0).

The majority of the medications reconciled were cardiovascular medications ( $n=144 ; 62.2 \%)$, followed by endocrinology medications ( $n=118 ; 51.1 \%$ ) and nutritional supplements and electrolytes $(n=69 ; 30 \%$ ) (Figure 1).

Table 1 below indicates the types of medication discrepancies upon hospital admission. One hundred and seventy eight cases $(76.8 \%)$ had medication discrepancies, with a median (IQR) average of medications discrepancies of $2.0(5.0,1.0)$. Most of the discrepancies were due to medication omissions ( $n=153 ; 66.1 \%)$, followed by incorrect dosages $(n=37 ; 16.3 \%)$ and different medications ( $n=30 ; 13.3 \%)$.

Clinical pharmacists' interventions were carried out in 150 cases (64.8\%) (Figure 2). There was a significant correlation between the number of clinical pharmacists' interventions and percentage of discrepancies (Spearman rho coefficient $=0.479 ; p<0.01$ ) .

Figure 2 above indicated that clinical pharmacists intervened the highest for omitted medications that required resumption ( $n=111$ of the cases; $44 \%$ ) followed

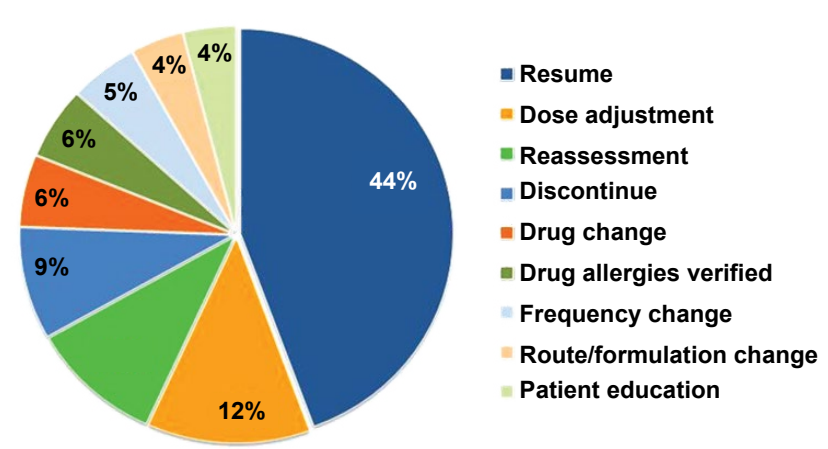

Figure 2: Percentages of clinical pharmacist interventions.

Table 2: Association between gender and level of medication discrepancies and level of omission.

\begin{tabular}{|c|c|c|c|c|}
\hline \multirow[t]{3}{*}{ Item } & \multirow[t]{3}{*}{ Number of medicines } & \multicolumn{2}{|c|}{ Gender } & \multirow[t]{3}{*}{ P value ${ }^{*}$} \\
\hline & & Male $(n=121)$ & Female $(n=111)$ & \\
\hline & & n (\%) & n (\%) & \\
\hline \multirow{3}{*}{$\begin{array}{l}\text { Level of medication } \\
\text { discrepancy }\end{array}$} & $0-2$ & $75(65)$ & $52(47)$ & \multirow[t]{3}{*}{0.157} \\
\hline & $3-5$ & $30(25)$ & $33(30)$ & \\
\hline & more than 5 & $16(13)$ & $26(23)$ & \\
\hline \multirow[t]{4}{*}{ Level of omission } & 0 & $42(35)$ & $37(33)$ & \multirow[t]{4}{*}{0.281} \\
\hline & $1-3$ & $56(46)$ & $45(41)$ & \\
\hline & $4-7$ & $17(14)$ & $27(24)$ & \\
\hline & 8-12 & $6(5)$ & $2(2)$ & \\
\hline
\end{tabular}

*Note: Analyses were done using Chi-Square test at alpha level $=0.05$. 
by medication dose adjustment $(n=31 ; 12 \%)$ and reassessment $(n=25 ; 10 \%)$.

Table 2 showed that there is a gender difference in terms of level of medication discrepancies but no difference was found with level of omission. Both male and females patients experienced higher medication discrepancies with lower number of medications (i.e. 0-2 medications is higher than 3-5 and more than 5) and the males had higher cases versus the females except for level of discrepancy of 3-5 and more than 5. Further analysis showed that gender is not a factor for number of medication discrepancies (Mann-Whitney $U$ test, $p=$ 0.157 ) and the rate of omission (Mann-Whitney $U$ test, $\mathrm{p}=0.281$ ).

\section{Discussion}

One of the missions of Al Wakra hospital - as the newest addition to HMC General Hospitals Group - is to provide safe and effective care for patients, which implies the importance of proper medication use. The clinical pharmacists acknowledged that potential harm could be caused by medication discrepancies. The activities of medication reconciliation aim to prevent discrepancies and potential medication-related problem. As reported in the literature, hospitals that implemented medication reconciliation may not experience the same impacts led to this required practice $[9,10]$.

\section{Number of medication reconciled and medication discrepancies}

There were more than 1500 medications reconciled. The highest number of medicines reconciled per patient was 17 and the majority was cardiovascular medications. Slightly more than three-forth of the cases had medication discrepancies. This was very worrisome. Furthermore, this study identified the most of the discrepancies were due to medication omissions. The overall rate of discrepancies was higher than some studies. Cornish and colleagues reported that eighty-one patients (53.6\%; 95\% confidence interval, $45.7 \%$ - 61.6\%) had at least one unintended discrepancy. The overall rate of unintended discrepancies was 0.93 per patient. The most common error (46.4\%) was omission of a regularly used medication and their findings were consistent with our study [11]. Cornu, et al. identified 681 discrepancies in 199 patients in their study with about three medications per patient [12]. According to them, around $82 \%$ of patients had at least one discrepancy. According to a review by Lo, et al. across thirteen medication reconciliation interventions, the median value for the number of clinically significant unintentional discrepancies per patient was 0.35 (Interquartile Range [IQR] 0.25-0.88) [10]. Medication reconciliation is a strategy to reduce this risk. This study did not determine the potential risk and seriousness of these discrepancies. Another issue with medication reconciliation concerns with the potential costs that might incur due to medication discrepan- cies. Nevertheless, this was not studied. Clinical pharmacists have proven their important roles through the interventions. According to Mueller, et al., key aspects of successful interventions included intensive pharmacy staff involvement and targeting the intervention to a high-risk patient population [4]. The number of medication discrepancy in our study is higher than the other studies.

\section{Association of med discrepancies and interventions}

Clinical pharmacists intervened in 150 cases with the highest for omitted medications that required resumption followed by medication dose adjustment and reassessment. Our study confirmed that the higher the number of clinical pharmacists' interventions, the higher would be the percentage of discrepancies detected. However, the study did not measure the percentage of interventions approved. Cornu, et al. documented $72 \%$ intervention approvals from 383 interventions that were carried out by the clinical pharmacists [12].

Implementation of a systematic medication reconciliation using a unified standard form served as a tool for detecting and resolving any medication discrepancies and resultant medication errors, as compared to physicians taking medication histories. The study results highlight the huge responsibility of clinical pharmacists in contribution to medication safety.

This preliminary study was conducted with few limitations. The study did not determine the number of accepted interventions did not classify the potential risk, did not take into consideration the cost factor that might be wasted due to medication discrepancies, and did not assess the outcome of the clinical pharmacy interventions.

\section{Study recommendation}

Findings from this study have shown that clinical pharmacists role in medication reconciliation is significant. Medication reconciliation will continue to be a required activity for all clinical pharmacists within $\mathrm{Al}$ Wakra hospital. Identifications of medication discrepancy areas will be used to target different populations with focus on high risk medications and high risk patients. Validating the results and MRF will make the researchers able to recommend to all hospitals under HMC to adopt their medication reconciliation method and MRF. This study is an essential part of ongoing process evaluation for quality improvement that falls under (Plan, Do, Study, Act) PDSA cycle. Medication reconciliation is a requirement for some organizations accreditation.

\section{Significance of the study findings}

There are few important points that could be implicated to practice:

I. Medication reconciliation is a mandatory role for all clinical pharmacists, as they are the safe guard against medication errors. 
II. Medication reconciliation is one of the key elements for effective communication between clinical pharmacists and physicians.

III. Medication reconciliation is a model for inter-professional collaborative care for patients, that is crucial for patient safety.

\section{Conclusions}

The study highlighted the importance of clinical pharmacists in conducting MR to prevent medication errors. Medication discrepancies upon hospital admission are highly common. The highest number of medicines reconciled per patient was 17 and the majority was cardiovascular medications. The highest number of medicines discrepancies was 2 and mostly were due to medication omissions. Clinical pharmacists intervened the highest for omitted medications that required resumption implementation of a systematic MR process using a standard form facilitated the detection and resolution of medication discrepancies.

\section{Conflict of Interests}

The author(s) declare that they have no competing interests.

\section{Acknowledgment}

The authors would like to thank the Medical Research Center of Hamad Medical Corporation and Qatar University IRB for approving the study.

\section{Funding}

This study was funded by Student Grant \#(QUST-CPHFALL-13/14-2) from the Qatar University. The statements made herein are solely the responsibility of the author(s).

\section{References}

1. (2013) ASHP statement on the pharmacist's role in medication reconciliation. Am J Health Syst Pharm 70: 453-456.
2. Coffey $M$, Cornish $P$, Koonthanam $T$, Etchells $E$, Matlow $A$ (2009) Implementation of admission medication reconciliation at two academic health sciences centres: Challenges and success factors. Healthc Q 12: 102-109.

3. Kwan JL, Lo L, Sampson M, Shojania KG (2013) Medication reconciliation during transitions of care as a patient safety strategy: A systematic review. Ann Intern Med 158: 397-403.

4. Mueller SK, Sponsler KC, Kripalani S, Schnipper JL (2012) Hospital-based medication reconciliation practices: A systematic review. Arch Intern Med 172: 1057-1069.

5. Barnsteiner JH (2008) Medication Reconciliation. In: Ronda G Hughes, Patient safety and quality: An evidence-based handbook for nurses. Agency for Healthcare Research and Quality, Rockville, USA, 1-14.

6. Gleason KM, McDaniel MR, Feinglass J, Baker DW, Lindquist $L$, et al. (2010) Results of the medications at transitions and clinical handoffs (MATCH) study: An analysis of medication reconciliation errors and risk factors at hospital admission. J Gen Intern Med 25: 441-447.

7. Nickerson A, MacKinnon NJ, Roberts N, Saulnier L (2005) Drug-therapy problems, inconsistencies and omissions identified during a medication reconciliation and seamless care service. Healthc $Q 8$.

8. JCAHO (2008) Medication Reconciliation Handbook. Joint Commission Resources, Inc. (JCR). Illinois, USA.

9. Kaboli PJ, Fernandes O (2012) Medication reconciliation: Moving forward. Arch Intern Med 172: 1069-1070.

10. Lo L, Kwan J, Fernandes OA, Shojania KG (2013) Medication reconciliation supported by clinical pharmacists (NEW). In: Making Health Care Safer II: An updated critical analysis of the evidence for patient safety practices. Agency for Healthcare Research and Quality, Rockville, USA.

11. Cornish PL, Knowles SR, Marchesano R, Tam V, Shadowitz $S$, et al. (2005) Unintended medication discrepancies at the time of hospital admission. Arch Intern Med 165: 424-429.

12. Cornu P, Steurbaut S, Leysen T, De Baere E, Ligneel C, et al. (2012) Effect of medication reconciliation at hospital admission on medication discrepancies during hospitalization and at discharge for geriatric patients. Ann Pharmacother 46: 484494. 